\title{
METALES PESADOS Y TOXICIDAD DE AGUAS DEL RÍO ACONCAGUA EN CHILE
}

\author{
Hernán Gaete*, Fernanda Aránguiz y Gabriela Cienfuegos \\ ${ }^{\mathrm{I}}$ Depto. Biología y Ciencias Ambientales, Facultad de Ciencias, Universidad de Valparaíso, Av. Gran Bretaña 1111, \\ Playa Ancha, Valparaíso - Chile \\ Marisol Tejos \\ Depto. Química y Bioquímica, Facultad de Ciencias, Universidad de Valparaíso, Av. Gran Bretaña 1111, Playa Ancha, \\ Valparaíso - Chile \\ Recebido em 12/5/06; aceito em 20/10/06; publicado na web em 28/5/07
}

\begin{abstract}
HEAVY METALS AND TOXICITY OF WATERS OF THE ACONCAGUA RIVER IN CHILE. The toxicity of surface waters in a zone with mining activity in the Aconcagua River was determined through growth inhibition bioassays of Pseudokirchneriella subcapitata, and correlated with heavy metal concentrations. Results show that the waters near the discharge of the mining effluent displayed toxicity during all periods of study; the molybdenum and copper concentration exceeded the norms of water quality. The correlations between the concentrations of metals and the growth rate of $P$. subcapitata varied in the different periods of the study; inverse and significant correlations with copper stand out in some periods.
\end{abstract}

Keywords: toxicity; Pseudokirchneriella subcapitata; heavy metals.

\section{INTRODUCCIÓN}

En la evaluación del riesgo ecológico en los ecosistemas acuáticos se considera la evaluación de la exposición y el efecto. Para la determinación del efecto se han propuesto métodos biológicos conocidos como bioensayos de toxicidad agudos y crónicos, con organismos representativos de diferentes niveles tróficos, los cuales dan una respuesta global a los contaminantes disueltos en las aguas superficiales que reciben la descarga de contaminantes químicos ${ }^{1,2}$.

En los ecosistemas acuáticos los organismos que pueden ser afectados por agentes químicos potencialmente tóxicos son los productores primarios, los cuales son claves en su estructura y funcionamiento, por lo que cualquier efecto negativo sobre ellos afectará los niveles tróficos superiores ${ }^{3}$. Algunas microalgas son consideradas modelos de estudio para la realización de bioensayos de toxicidad, debido a que presentan alta sensibilidad a diversas sustancias químicas, sus requerimientos nutricionales son conocidos, poseen una alta tasa de crecimiento que permite conocer en pocos días la densidad y el efecto causado por el agente tóxico y su manipulación es relativamente sencilla en laboratorio ${ }^{4}$.

Los metales pesados se destacan por sus efectos tóxicos sobre los organismos acuáticos, los cuales pueden provenir de fuentes naturales o antropogénicas ${ }^{5}$. El cobre es un elemento esencial, requerido por todas las plantas y animales, incluyendo la vida acuática ya que forma parte de una variedad de enzimas que juegan un rol importante en procesos fisiológicos, así como en algunas proteínas estructurales $^{5-7}$, pero a altas concentraciones el cobre se vuelve una sustancia tóxica ${ }^{5}$. Las fuentes antropogénicas de contaminación ambiental por cobre incluyen a la minería, fundiciones, y una variedad de productos basados en cobre utilizados en construcción, equipamientos electrónicos y en otras industrias ${ }^{6}$.

Un importante componente de la exposición de un organismo a un metal es la biodisponibilidad - fracción del metal total que esta disponible para ejercer acción y efecto en el organismo receptor ${ }^{2}$.

*e-mail: Hernan.Gaete@uv.cl
La toxicidad del cobre se relaciona principalmente con iones libres y ésta toxicidad varía entre especies de microalgas. Las formas de cobre libre no son las predominantes, sino que son los complejos orgánicos y éstos no son biodisponibles para las algas ${ }^{8}$.

En las aguas dulces, el pH y la formación de complejos orgánicos son importantes en determinar la especiación, biodisponibilidad y toxicidad del cobre ${ }^{2,9-11}$. Además, la variabilidad de la actividad del ión hidrogeno es un factor que altera la especiación y la concentración de las formas tóxicas del cobre. Se ha demostrado que componentes orgánicos pueden reducir la toxicidad del cobre y de otros metales sobre los organismos?.

En la cuenca del río Aconcagua (V Región, Chile Central), se desarrolla una intensa actividad minera. Esto ha provocado un aumento de los niveles de metales pesados en el río, especialmente el cobre $^{12}$. Esta alteración de la calidad de sus aguas podría estar confiriendo características de toxicidad a estas aguas superficiales. Por este motivo, en esta investigación se planteó como objetivo determinar la toxicidad de las aguas superficiales del río Aconcagua en una zona con actividad minera y correlacionar la tasa de crecimiento de Pseudokirchneriella subcapitata con las concentraciones de metales pesados especialmente cobre.

\section{MATERIALES Y MÉTODOS}

\section{Muestreo}

Las muestras de agua para análisis fueron tomadas en recipientes plásticos en siete puntos de muestreo a lo largo del río Aconcagua en noviembre de 2004, mayo, julio y septiembre de 2005, de acuerdo a la norma NCh411/6.Of98, Calidad del agua Muestreo ${ }^{13}$ (Figura 1). Las muestras fueron transportadas refrigeradas $\left(4^{\circ} \mathrm{C}\right)$ a la Universidad de Valparaíso para su posterior análisis químico y realización del bioensayo de toxicidad.

La determinación de $\mathrm{pH}$, conductividad, temperatura y sólidos disueltos totales (SDT) se realizó in situ con un equipo multiparámetro Hanna HI 98130, y la determinación de las variables oxígeno disuelto (OD) y porcentaje de saturación de oxígeno se 
realizó con un equipo LABCOR Consort C534. Los niveles de nitrato $\left(\mathrm{NO}_{3}-\mathrm{N}\right)$ y fósforo total se determinaron a través de colorimetría con un equipo Orbeco - Hellige $975 \mathrm{MP}^{14}$.

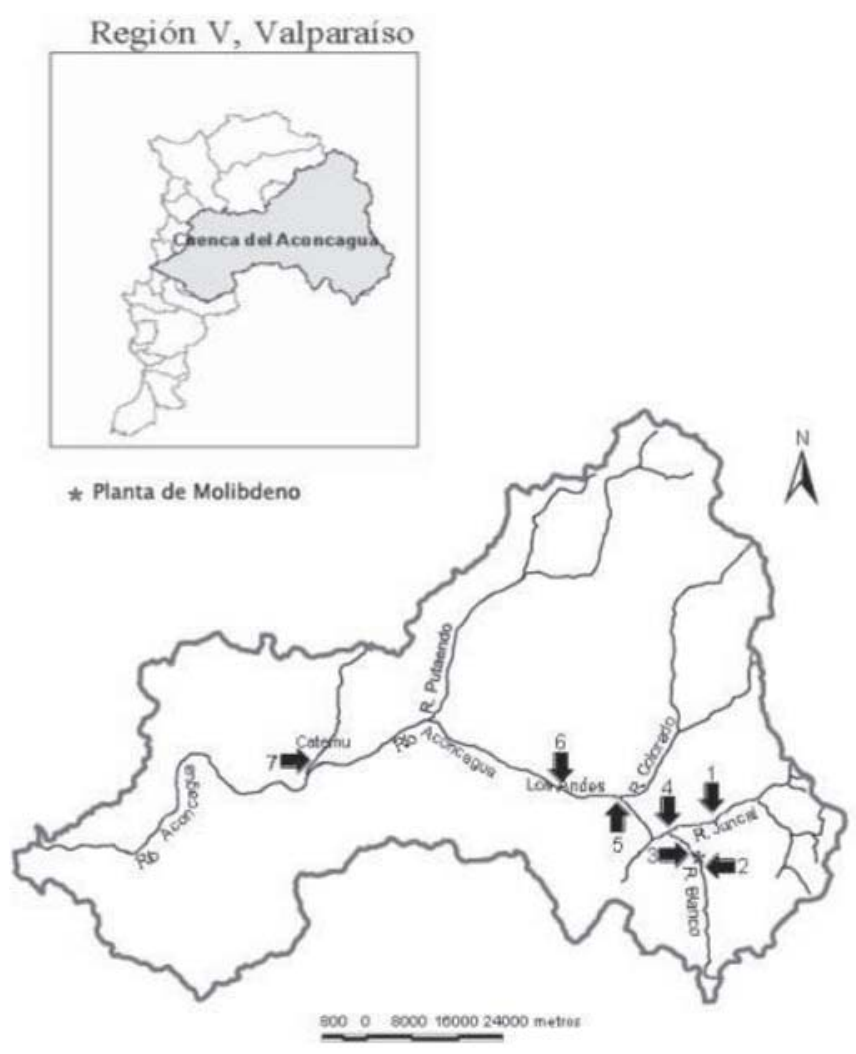

Figura 1. Localización de los puntos de muestreo en la zona de estudio (río Aconcagua)

\section{Análisis químico}

Los análisis químicos de los metales cobre (disuelto y total), molibdeno total, zinc total, hierro total y dureza $\left(\mathrm{CaCO}_{2}\right)$ se determinaron por espectrofotometría de absorción atómica en horno de grafito, para determinar la porción disuelta del cobre las muestras fueron filtradas con filtro de membrana de $0,45 \mu \mathrm{m}^{7,11}$. Todos estos análisis se realizaron en el Laboratorio de Toxicología Humana y Ambiental de la Universidad de Playa Ancha y en el Laboratorio de Química Analítica de la Facultad de Ciencias de la Universidad de Valparaíso.

\section{Bioensayos}

Como organismo de prueba, se utilizó la microalga unicelular de agua dulce Pseudokirchrneriella subcapitata, perteneciente al orden Chlorococcales clase Chlorophyceae, división Chlorophyta. La cepa fue adquirida en el Laboratorio de Ficología de la Universidad de Concepción. Los bioensayos fueron realizados de acuerdo con el procedimiento a la norma NCh2709/Of2002 ${ }^{15}$.

Previo a cada bioensayo se prepararon las soluciones de nutrientes compuestas por Fe-EDTA, micronutrientes: B, Mn, Zn, Co, Mo, y macronutrientes: N, P, Mg, Ca, S, K, Na. Luego, en matraces Erlenmeyer, se inocularon las muestras de agua de río con un mismo volumen de Pseudokirchneriella subcapitata de un medio de cultivo que se encontraba en fase exponencial de crecimiento y fueron enriquecidas con una misma concentración de nutrientes para evitar falsos negativos debido a la deficiencia de nutrientes. Adicio- nalmente una solución control preparada con agua destilada, reemplazando el agua de río, fue enriquecida con los mismos nutrientes. La concentración celular inicial fue de 100.000 céls $/ \mathrm{mL}$. Cada una de las muestras y control tuvieron cuatro réplicas. Los tratamientos resultantes con volumen de $50 \mathrm{~mL}$ se mantuvieron bajo luz blanca fría continua con una intensidad lumínica de $90 \pm 10 \mu \mathrm{mol} \mathrm{m} \mathrm{m}^{-2} \mathrm{~s}^{-1}$, a una temperatura de $23 \pm 2{ }^{\circ} \mathrm{C}$ y con agitación manual dos veces al día.

La densidad celular $(\mathrm{N})$ se determinó cada 24 h por conteo directo en microscopio utilizando cámara de Neubauer de línea brillante durante $96 \mathrm{~h}$ y se determinó la tasa de crecimiento $\mathrm{k}$ en número de duplicaciones celulares por día (duplic/día) a partir de

$k=3,322 \frac{\log N_{n}-\log N_{0}}{t_{n}}$,

donde $N_{n}$ es la densidad celular al final del bioensayo; $N_{0}$ es la densidad celular inicial nominal y $t_{n}$ es el tiempo transcurrido entre el inicio y final del bioensayo (en días). También se determinó el porcentaje de inhibición de la tasa de crecimiento $\left(\mathrm{I}_{\mathrm{k}}\right)$

$I_{k i}=\underline{k_{c}-\underline{k_{i}}} \underline{k_{c}} \times 100$

en donde $k_{c}$ es la tasa de crecimiento promedio para el control; y $k_{i}$ corresponde a la tasa de crecimiento promedio para la concentración $i^{15}$. La correlación entre variables se determinó por análisis de Pearson y la significancia de la diferencia entre medias se realizó mediante la prueba T de Student. Para ello se utilizó el programa Systat.

\section{RESULTADOS Y DISCUSIÓN}

La Tabla 1 presenta los parámetros fisicoquímicos determinados en el río Aconcagua. Estos resultados muestran que la estación 3 presentó los niveles más bajos de $\mathrm{pH}$ y altos niveles de conductividad y sólidos disueltos totales en noviembre, mayo y septiembre en comparación a las otras estaciones, mientras que en julio esta situación cambió lo que podría deberse a que durante ese muestreo el efluente minero de la Planta de Molibdeno (Figura 1) cercano a dicha estación presentaba una notoria disminución de su caudal.

$\mathrm{El} \mathrm{pH}$ fue levemente alcalino en todas las estaciones durante todos los períodos de estudio situándose en el rango establecido por la NCh1333Of $78^{16}$ para aguas destinadas a riego. Estos niveles son similares a los medidos en la zona de estudio por la Dirección General de Aguas (DGA) el año 2004 ${ }^{12}$.

Similar pH se determinó en estudios realizados en el norte de Chile en el río Elqui (IV Región), y más al sur (VI Región) en el río Cachapoal, en los cuales se estudió el efecto de las explotaciones mineras sobre la calidad de sus aguas. En ambos estudios se determinaron los niveles más bajos de $\mathrm{pH}$ aguas abajo de efluentes mineros y niveles mas elevados en aguas que no reciben directamente las descargas mineras ${ }^{17,18}$, sin embargo, en el río Elqui se determinaron niveles aún mas bajos en comparación al presente estudio, lo cual puede deberse a diferencias en la actividad productiva de las empresas mineras, así como a las diferencias de caudal de los dos ríos, ya que los ríos mas caudalosos y rápidos tienen una capacidad de dilución mayor.

Los niveles de conductividad determinados en la estación 3 en noviembre, mayo y septiembre corresponden según la NCh1333Of $78^{16}$ a aguas que puede tener efectos adversos sobre cultivos. Estos niveles de conductividad son más elevados a los determinados en otros estudios en el mismo sector ${ }^{12}$. Niveles más elevados se determinaron en el estudio de Guevara ${ }^{17}$ en las aguas cercanas al 
Tabla 1. Parámetros fisicoquímicos determinados en los periodos de estudio.

\begin{tabular}{|c|c|c|c|c|c|c|c|c|}
\hline \multirow[t]{2}{*}{ Parámetro } & \multirow[t]{2}{*}{ Muestreo } & \multicolumn{7}{|c|}{ Estación } \\
\hline & & 1 & 2 & 3 & 4 & 5 & 6 & 7 \\
\hline \multirow[t]{4}{*}{ Oxígeno Disuelto [mg/L] } & Noviembre & 11,1 & 10,4 & 9,5 & 10,1 & 8,8 & 7,3 & 8,8 \\
\hline & Mayo & 10,3 & 10,0 & 10,9 & 10,4 & 10,1 & 10,6 & 10,5 \\
\hline & Julio & 12,5 & 12,1 & 12,1 & 11,8 & 12,7 & 12,0 & 12,1 \\
\hline & Septiembre & 11,8 & 7,4 & 7,6 & 7,35 & 10,6 & 10,1 & 10,0 \\
\hline \multirow[t]{4}{*}{ \% Saturación de Oxigeno } & Noviembre & 102,0 & 97,0 & 96,5 & 96,5 & 92,5 & 75,0 & 99,0 \\
\hline & Mayo & 100,0 & 99,0 & 97,0 & 99,0 & 97,0 & 96,5 & 102,0 \\
\hline & Julio & 104,0 & 112,0 & 94,5 & 96,5 & 96,5 & 96,5 & 114,0 \\
\hline & Septiembre & 102,0 & 114,0 & 73,0 & 67,5 & 100,0 & 94,5 & 112,0 \\
\hline \multirow[t]{4}{*}{$\mathrm{pH}$} & Noviembre & 8,35 & 8,34 & 8,03 & 8,26 & 8,37 & 8,06 & 8,08 \\
\hline & Mayo & 8,24 & 8,11 & 7,20 & 8,08 & 8,15 & 7,93 & 8,13 \\
\hline & Julio & 8,27 & 8,10 & 7,90 & 8,10 & 8,00 & 8,00 & 8,30 \\
\hline & Septiembre & 8,18 & 7,90 & 7,50 & 7,88 & 7,87 & 7,80 & 8,26 \\
\hline \multirow[t]{4}{*}{ Conductividad $[\mathrm{mS} / \mathrm{cm}]$} & Noviembre & 0,32 & 0,19 & 1,80 & 0,29 & 0,14 & 0,26 & 0,53 \\
\hline & Mayo & 0,52 & 0,40 & 1,80 & 0,60 & 0,27 & 0,48 & 0,52 \\
\hline & Julio & 0,44 & 0,30 & 0,37 & 0,29 & 0,20 & 0,37 & 0,53 \\
\hline & Septiembre & 0,36 & 0,21 & 1,50 & 0,25 & 0,16 & 0,25 & 0,48 \\
\hline \multirow[t]{4}{*}{ Sólidos Disueltos Totales [mg/L] } & Noviembre & 160 & 90 & 900 & 140 & 60 & 140 & 270 \\
\hline & Mayo & 270 & 230 & 1230 & 330 & 150 & 260 & 280 \\
\hline & Julio & 210 & 140 & 180 & 150 & 100 & 170 & 270 \\
\hline & Septiembre & 180 & 110 & 530 & 120 & 80 & 120 & 240 \\
\hline \multirow[t]{4}{*}{$\mathrm{t}\left[{ }^{\circ} \mathrm{C}\right]$} & Noviembre & 8,8 & 10,7 & 13,9 & 13,5 & 15,5 & 13,0 & 18,9 \\
\hline & Mayo & 5,9 & 9,0 & 12,0 & 12,6 & 14,6 & 13,6 & 18,4 \\
\hline & Julio & 9,5 & 9,7 & 10,7 & 11,0 & 10,7 & 10,7 & 17,0 \\
\hline & Septiembre & 9,2 & 11,6 & 13,0 & 11,4 & 11,2 & 12,0 & 10,0 \\
\hline \multirow[t]{4}{*}{ Dureza $\left[\mathrm{mg} \mathrm{CaCO}_{3} / \mathrm{L}\right]$} & Noviembre & 325,0 & 188,0 & 361,0 & 184,0 & 125,0 & 264,0 & 526,0 \\
\hline & Mayo & 238,0 & 187,0 & 138,0 & 86,1 & 64,8 & 124,0 & 323,0 \\
\hline & Julio & 158,0 & 85,1 & 70,8 & 58,2 & 26,7 & 60,7 & 196,0 \\
\hline & Septiembre & 166,0 & 89,6 & 130,0 & 92,1 & 67,7 & 98,6 & 208,0 \\
\hline \multirow[t]{4}{*}{ Nitratos $[\mathrm{mg} / \mathrm{L}]$} & Noviembre & 0,14 & 0,11 & 0,05 & 0,28 & 0,09 & 0,18 & 4,00 \\
\hline & Mayo & 0,28 & 0,19 & 0,09 & 0,25 & 0,32 & 0,38 & 2,90 \\
\hline & Julio & 0,37 & 0,27 & 0,19 & 0,27 & 0,45 & 0,49 & 2,40 \\
\hline & Septiembre & 0,39 & 0,32 & 0,28 & 0,34 & 0,13 & 0,13 & 3,40 \\
\hline \multirow[t]{4}{*}{ Fósforo total[mg/L] } & Noviembre & 4,49 & 3,32 & 2,68 & 2,82 & 6,47 & 17,06 & 36,63 \\
\hline & Mayo & 5,63 & 5,23 & 12,11 & 8,96 & 7,60 & 9,61 & 9,06 \\
\hline & Julio & 7,72 & 5,34 & 6,20 & 6,05 & 10,69 & 9,70 & 4,76 \\
\hline & Septiembre & 5,04 & 3,30 & 14,12 & 7,66 & 6,60 & 7,46 & 7,19 \\
\hline
\end{tabular}

distrito minero de El Indio, esta diferencia con el presente estudio puede deberse a diferencias en el proceso productivo y en los tratamientos de residuos líquidos de las empresas mineras y a que ambos ríos presentan diferentes caudales durante el año.

Similares concentraciones de sólidos disueltos totales del presente estudio se determinaron en mediciones anteriores realizadas en el río Aconcagua, entre septiembre de 1998 y julio de $1999^{19}$.

Las concentraciones de oxigeno disuelto no presentaron mayores variaciones en los periodos de estudio. Las concentraciones mínimas determinadas en este estudio son más bajas a las determinadas en períodos anteriores ${ }^{12}$. Cabe destacar que los niveles de oxígeno disuelto en todas las estaciones estuvieron sobre la concentración mínima de $5 \mathrm{mg} / \mathrm{L}$ que establece la normativa chilena para la protección de la vida acuática ${ }^{16}$, mientras que los porcentajes de saturación de oxígeno estuvieron sobre el 70\%. En general, la zona de estudio corresponde a ritrones lo que favorece la aireación de las aguas y también su menor temperatura en la zona cordillerana.

La temperatura tuvo un comportamiento similar durante todo el estudio, siendo aguas arriba (estación 1) donde se encontraron las temperaturas más bajas y en la estación 7 las temperaturas más altas, excepto en septiembre, donde la temperatura en dicha estación es notoriamente más baja que la registrada en los otros muestreos.

En cuanto a la dureza, durante todos los periodos de estudio se encontraron los niveles más bajos en la estación 5, correspondiendo, según la clasificación de Romero ${ }^{20}$, a aguas blandas o moderadamente duras, y los más altos en la estación 7, correspondiendo a aguas duras o muy duras. En general, durante todos los muestreos, los niveles se encuentran dentro de la NCh1333Of 78 para aguas destinadas a la vida acuática ${ }^{16}$.

No se encontró una tendencia espacial ni temporal en las concentraciones de fósforo, mientras que los niveles de nitrato son relativamente bajos en la mayoría de las estaciones, excepto en la estación 7, observándose esta tendencia en todos los períodos de estudio. En la estación 7 se observó importante cantidad de vegetación, lo que estaría indicando un proceso de eutrofización. Este aumento del nitrógeno podría provenir del uso intensivo de fertilizantes en las actividades agrícolas que allí se desarrollan. A excepción de la estación 7, los niveles de nitrato fueron menores a los determinados en años anteriores en el mismo sector ${ }^{21}$.

Las concentraciones de zinc total no exhibieron un patrón espacial ni temporal definido (Figura 2a), y no se superó la norma chilena de $30,57 \mu \mathrm{mol} / \mathrm{L}$ para aguas destinadas a riego ${ }^{16}$. Los niveles determina- 
dos son similares a los encontrados por la DGA en el año $2004^{12}$. Lo anterior sugiere la ausencia de cambios significativos en las fuentes de descarga de este metal en el río Aconcagua entre el período de muestreo de dicho análisis y la fecha de muestreo de este estudio.

Altas concentraciones de molibdeno total se determinaron en noviembre, mayo y septiembre en la estación 3, lo que disminuyó considerablemente en julio (Figura 2b). La norma chilena para aguas destinadas a riego de $0,10 \mu \mathrm{mol} / \mathrm{L}^{16}$ se superó en varias estaciones y periodos de estudio. La disminución de las concentraciones de molibdeno en julio puede deberse a la disminución del caudal de las descargas de la Planta de Molibdeno, observada en terreno. Las concentraciones de molibdeno de este estudio son similares a las determinadas por la DGA en el mismo sector el año 2004 ${ }^{12}$. Las altas concentraciones de molibdeno determinadas en la estación 3 durante el presente estudio sugieren el fuerte impacto del efluente de la Planta de Molibdeno, única fuente de descarga en el sector, ubicada aguas arriba de este punto de muestreo, sin embargo, concentraciones sobre la norma en la estación 1, que no se encuentra próxima a descargas mineras, puede indicar la presencia de fuentes naturales del metal.

Se determinaron bajas concentraciones de hierro total (Figura 2c) en comparación a la norma chilena para aguas destinada a riego y a los niveles determinados en el estudio de la DGA del año $2004^{12}$ en el mismo sector.

Altas concentraciones de cobre total se determinaron en la estación 3 y 6 en noviembre y septiembre y sólo en la estación 3 en mayo, superando la NCh1333 para aguas destinadas a riego ${ }^{16}$, mientras que en julio los niveles fueron más bajos (Figura 2d). Las altas concentraciones determinadas en la estación 3 podrían reflejar la descarga del metal atribuible a las actividades mineras del sector (Planta de Molibdeno). La disminución de la concentración en la estación 3 en julio, coincidió con la notoria disminución de la descarga del efluente minero cercano a esta estación. Concentraciones superiores de cobre total se determinaron en el año 2004 en el mismo sector por la DGA ${ }^{12}$.

En los estudios de Guevara ${ }^{17}$ y Richtera ${ }^{18}$, realizados en el norte y sur de Chile, respectivamente, se determinaron concentraciones más altas de cobre total a las determinadas en el presente estudio, esta diferencia se explicaría debido a variaciones en los procesos productivos de las empresas mineras, en los tratamientos de los residuos líquidos generados, así como en diferencias de caudal de los ríos.

Concentraciones sobre los $2,83 \mu \mathrm{mol} / \mathrm{L}$ de cobre disuelto se determinaron en la estación 3 en noviembre, mayo y septiembre (Figura 2e), concentraciones que representan más del 60\% del cobre total. Similar porcentaje representa el cobre disuelto del total del metal en el estudio de Kramer et al. ${ }^{11}$, sin embrago, las concentraciones de este metal son más bajas en dicho estudio.

Debido a que muchos parámetros como $\mathrm{pH}$, conductividad, sólidos disueltos y las concentraciones de los metales pesados, especialmente cobre total y disuelto, molibdeno y zinc totales, varían considerablemente en la estación 3, con respecto de las otras estaciones, se analizó físico químicamente, en agosto del 2006, el efluente minero de la Planta de Molibdeno, el cual sería el responsable de esta variación, los resultados obtenidos se muestran en la Tabla 2.

Los resultados obtenidos del análisis fisicoquímico realizado al efluente de la planta de molibdeno evidencian el impacto que genera la actividad minera sobre la calidad de las aguas en el sector de la estación 3.

Un factor importante que puede influir en la variación de los niveles de parámetros físico-químicos y en la concentración total de metales pesados es el caudal del río ${ }^{17}$. El río Aconcagua presenta sus mayores caudales entre noviembre y marzo y los menores entre junio
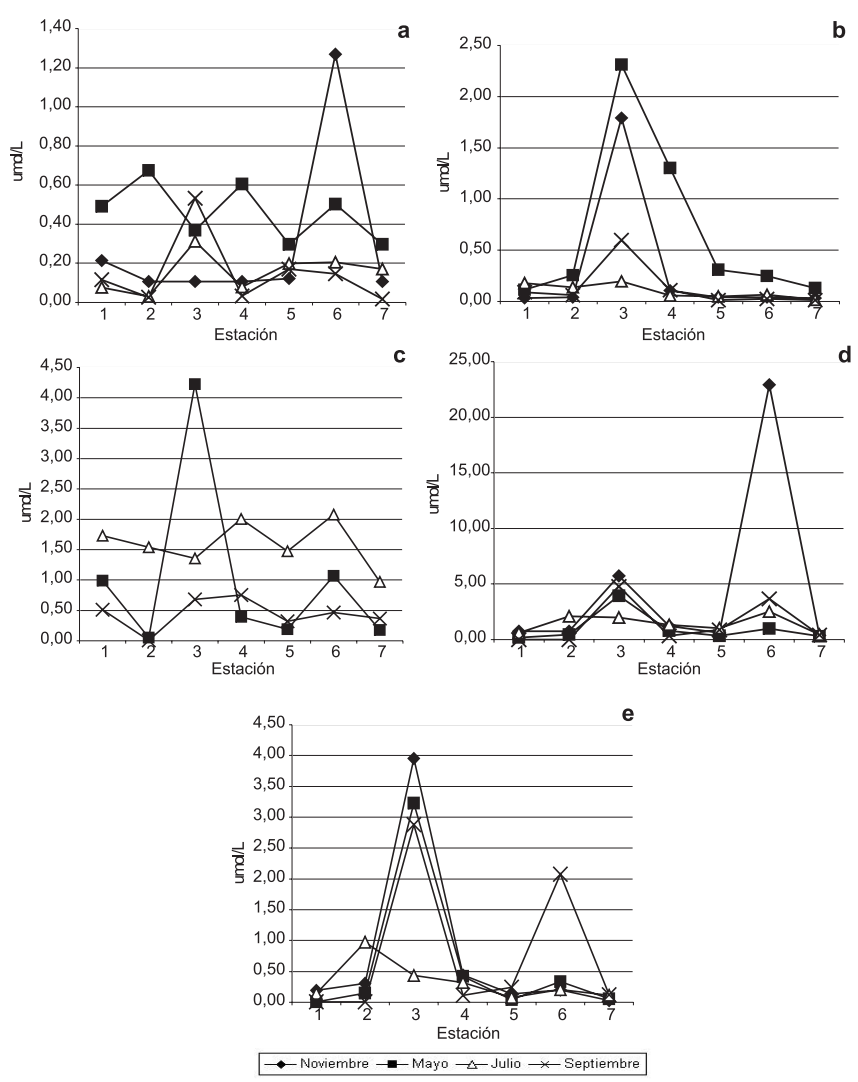

Figura 2. Variación temporal y espacial de la concentración de metales: zinc total (a), molibdeno total (b), hierro total (c), cobre total (d) y cobre disuelto $(e)$

Tabla 2. Análisis fisicoquímico del efluente minero de la Planta de Molibdeno

\begin{tabular}{lc}
\hline Parámetro & \\
\hline $\mathrm{pH}$ & 7,07 \\
Conductividad $[\mathrm{mS} / \mathrm{cm}]$ & 3,24 \\
Sólidos disueltos totales $[\mathrm{mg} / \mathrm{L}]$ & 1610 \\
Temperatura $\left[{ }^{\circ} \mathrm{C}\right]$ & 15,2 \\
$\mathrm{Cu}$ total $[\mu \mathrm{mol} / \mathrm{L}]$ & 26,09 \\
$\mathrm{Cu}$ disuelto $[\mu \mathrm{mol} / \mathrm{L}]$ & 12,56 \\
Zinc total $[\mu \mathrm{mol} / \mathrm{L}]$ & 120,78 \\
Molibdeno total $[\mu \mathrm{mol} / \mathrm{L}]$ & 40,58 \\
Hierro total $[\mu \mathrm{mol} / \mathrm{L}]$ & 35,28 \\
\hline
\end{tabular}

y septiembre ${ }^{12}$, estas variaciones pueden estar asociadas también al régimen mixto del río ${ }^{21}$. Además, en las estaciones 4,5 y 6 el caudal del río aumenta considerablemente (Figura 3), lo que podría favorecer la dilución de los metales, debido principalmente al aporte de los ríos Juncal y Colorado (Figura 1), disminuyendo su concentración en las aguas del Aconcagua, lo que no se aplica a la estación 7 debido a que se encuentra en un afluente del Aconcagua (Figura 1). Otro factor importante que podría influir en la variación temporal de la concentración de los metales pesados y algunos parámetros fisicoquímicos, y que podría influir en los resultados de julio, son las variaciones temporales de la actividad productiva de las empresas mineras ubicadas en el sector estudiado.

En las Figuras 4 y 5 se observan efectos tanto inhibitorios como estimuladores de las muestras sobre el crecimiento de la microalga con respecto al control de laboratorio. En todos los periodos de 


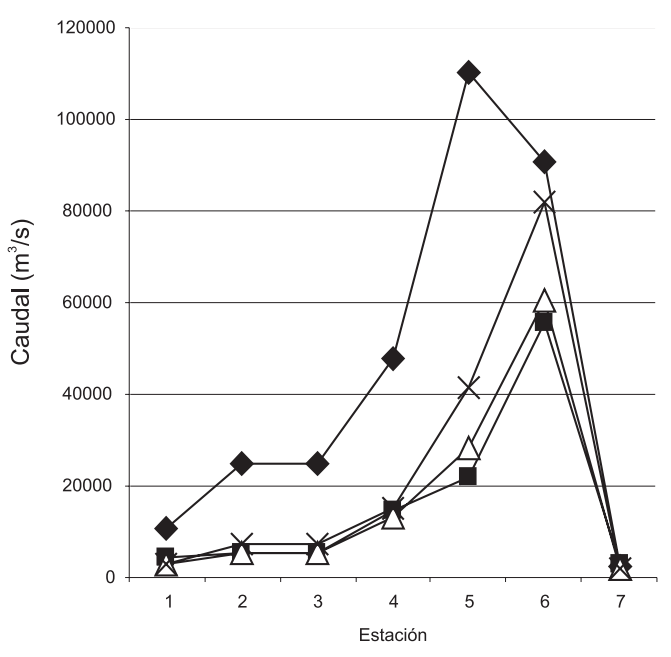

Noviembre - - Mayo $\triangle-$ Julio $\rightarrow$ Septiembre

Figura 3. Caudal mensual en las estaciones de muestreo. Fuente: Dirección General de Aguas

estudio se observó un menor crecimiento celular en las estaciones 3 y 6 , lo que puede deberse a que en las estaciones 3 y 6 se determinaron los niveles más bajos de $\mathrm{pH}$ y mas altos de conductividad y concentración de sólidos disueltos totales así como altas concentraciones de metales pesados debido a que se encuentran próximas a fuentes de contaminación como efluentes mineros y descargas de aguas domiciliarias e industriales.
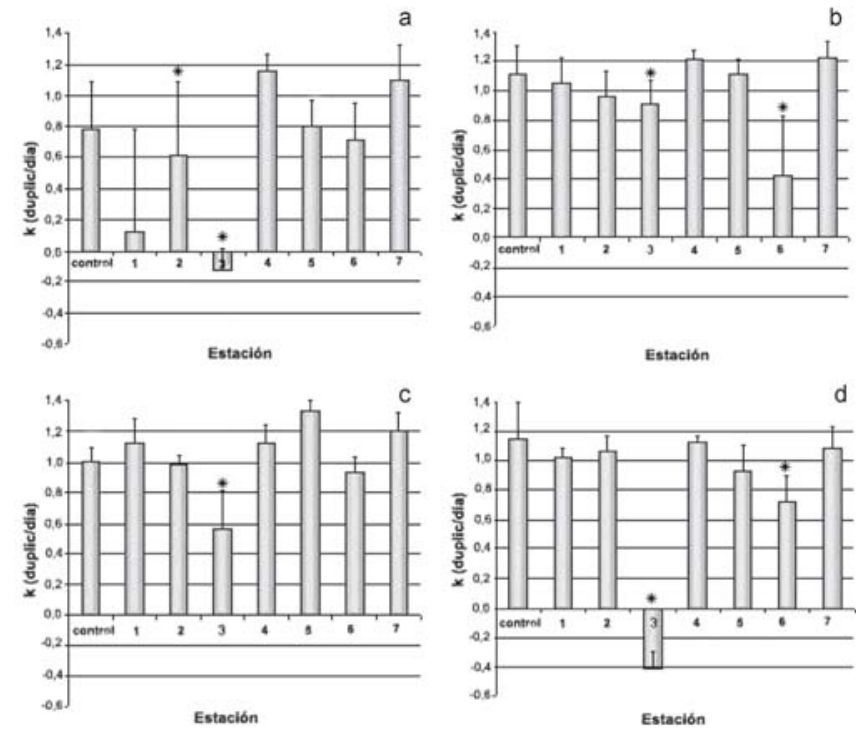

Figura 4. Tasa de crecimiento a las 96 h para P. subcapitata durante los bioensayos: noviembre (a), mayo (b), julio $(c)$ y septiembre $(d)$. *: Indica diferencia significativa con la estación 7

La estación 4 presentó estimulación del crecimiento con respecto al control en todos los períodos de estudio (Figura 5), lo que se explicaría por el efecto de dilución provocada por la confluencia del río Blanco con el río Juncal (Figura 1), y que se vio reflejado, en comparación a la estación 3, en aumento del pH, disminución de la conductividad y concentración de sólidos disueltos totales y en la disminución de las concentraciones de cobre disuelto y total y molibdeno (Tabla 1 y Figura 2), disminuyendo los potenciales efectos tóxicos de agentes químicos como el cobre sobre la microalga. El comportamiento de la microalga en la estación 4 podría ser además, el resultado de la estimulación que se produce en bajas concentraciones de cobre ${ }^{22}$.

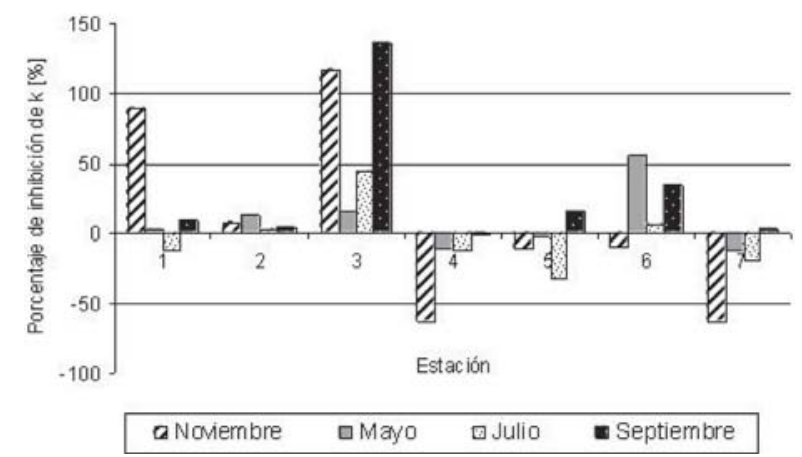

Figura 5. Porcentaje de inhibición de la tasa de crecimiento $k$ a las 96 h con respecto al control de laboratorio en los bioensayos

En la Tabla 3 se muestran las correlaciones entre metales pesados y la tasa de crecimiento de $P$. subcapitata, destacándose en noviembre y mayo la significativa correlación entre las concentraciones de molibdeno y cobre disuelto y en septiembre entre el zinc, cobre disuelto y molibdeno, lo que sugiere que estos metales provendrían de la misma fuente de descarga. Cabe destacar que en julio no se correlacionaron significativamente los metales estudiados, lo que podría deberse a la disminución de las descargas mineras, sumado al aumento de las temperaturas por la llegada de la primavera que trae consigo los deshielos y por consiguiente el aumento del caudal del río (Figura 3), favoreciendo la dilución de los metales estudiados.

Noviembre es el mes con mayores caudales (Figura 3), sin embargo, la estación 1 es la que presenta menores variaciones de caudal respecto de las demás estaciones, por lo que si bien la variación de caudal es importante para la dilución en las demás estaciones, en la estación 1 no representa mayores efectos.

La relación inversa en septiembre entre la tasa de crecimiento (k) y el cobre disuelto, cobre total, zinc y molibdeno (Tabla 3) puede indicar que las concentraciones de estos metales pesados durante este período de estudio serían las que provocaron la toxicidad de las aguas que ocasionó la inhibición de la tasa de crecimiento de las microalgas durante el bioensayo.

En la Figura 4a se observa que en la estación 1 en noviembre se produjo una baja tasa de crecimiento, no presentando diferencias significativas con la estación 3. Este punto es de especial interés, dado que la estación 1 no se encuentra afectada por la actividad minera de la zona, lo cual sugiere la existencia de otros agentes químicos no considerados en este estudio que provocaron efectos tóxicos. La estación 3 en todos los períodos presentó inhibición del crecimiento con respecto al control de laboratorio (Figuras 5), especialmente en los meses de noviembre y septiembre, coincidiendo con los niveles mas bajos de $\mathrm{pH}$, más altos niveles de conductividad y concentración de sólidos disueltos totales y las concentraciones más altas de metales pesados (Tabla 1 y Figura 2). Además, durante todos los periodos de estudio la estación 3 presentó diferencias significativas con respecto a la estación 7 , establecida como estación control debido a que se encuentra en un afluente del río Aconcagua, no se ve afectada por la actividad minera de la zona y porque no presentó mayores variaciones en la tasa de crecimiento a las 96 h (Figura 4).

Debido a que la estación 3 presentó elevados porcentajes de inhibición del crecimiento con respecto al control de laboratorio 
Tabla 3. Correlaciones entre $\mathrm{pH}$, tasa de crecimiento (k) y metales pesados

\begin{tabular}{|c|c|c|c|c|c|c|c|c|}
\hline & Bioensayo & $\mathrm{k}$ & $\mathrm{Cu}$ disuelto & $\mathrm{Cu}$ total & $\mathrm{Zn}$ & Mo & $\mathrm{Fe}$ & $\mathrm{pH}$ \\
\hline \multirow[t]{4}{*}{$\mathrm{Cu}$ disuelto } & Noviembre & 0,600 & 1,000 & & & & & \\
\hline & Mayo & $-0,167$ & 1,000 & & & & & \\
\hline & Julio & $-0,438$ & 1,000 & & & & & \\
\hline & Septiembre & $-0,899 *$ & 1,000 & & & & & \\
\hline \multirow[t]{4}{*}{$\mathrm{Cu}$ total } & Noviembre & $-0,112$ & 0,057 & 1,000 & & & & \\
\hline & Мауо & $-0,248$ & $0,995 *$ & 1,000 & & & & \\
\hline & Julio & $-0,656$ & 0,563 & 1,000 & & & & \\
\hline & Septiembre & $-0,883 *$ & $0,996^{*}$ & 1,000 & & & & \\
\hline \multirow[t]{4}{*}{ Zn total } & Noviembre & $-0,016$ & $-0,192$ & $0,965^{*}$ & 1,000 & & & \\
\hline & Mayo & $-0,187$ & $-0,202$ & $-0,180$ & 1,000 & & & \\
\hline & Julio & $-0,485$ & $-0,376$ & 0,208 & 1,000 & & & \\
\hline & Septiembre & $-0,976^{*}$ & $0,838^{*}$ & $0,830 *$ & 1,000 & & & \\
\hline \multirow[t]{4}{*}{ Mo total } & Noviembre & $-0,604$ & $0,998 *$ & 0,056 & $-0,192$ & 1,000 & & \\
\hline & Mayo & 0,046 & $0,911^{*}$ & $0,903 *$ & $-0,044$ & 1,000 & & \\
\hline & Julio & $-0,670$ & 0,419 & 0,251 & 0,058 & 1,000 & & \\
\hline & Septiembre & $-0,928^{*}$ & 0,723 & 0,687 & $0,901 *$ & 1,000 & & \\
\hline \multirow[t]{4}{*}{ Fe total } & Noviembre & - & - & - & - & - & - & \\
\hline & Mayo & $-0,289$ & $0,961^{*}$ & $0,959^{*}$ & $-0,239$ & $0,816^{*}$ & 1,000 & \\
\hline & Julio & $-0,043$ & 0,025 & 0,476 & $-0,275$ & 0,069 & 1,000 & \\
\hline & Septiembre & $-0,405$ & 0,405 & 0,403 & 0,423 & 0,489 & 1,000 & \\
\hline \multirow[t]{4}{*}{$\mathrm{pH}$} & Noviembre & 0,119 & $-0,507$ & $-0,567$ & $-0,410$ & $-0,532$ & - & 1,000 \\
\hline & Mayo & 0,309 & $-0,983 *$ & $-0,994 *$ & 0,211 & $-0,865^{*}$ & $-0,946^{*}$ & 1,000 \\
\hline & Julio & 0,561 & $-0,219$ & $-0,737$ & $-0,599$ & $-0,232$ & $-0,271$ & 1,000 \\
\hline & Septiembre & $0,787 *$ & $-0,779 *$ & $-0,779 *$ & $-0,768^{*}$ & $-0,717$ & $-0,286$ & 1,000 \\
\hline
\end{tabular}

*: Indica correlación significativa $(\mathrm{p}<0,05)$

(Figura 5) se realizó en los meses de noviembre y septiembre bioensayos con diluciones, obteniéndose en el mes de noviembre un valor de $\mathrm{IC}_{50}=18,91 \%$ y en el mes de septiembre un valor de $\mathrm{IC}_{50}=17,99 \%$.

Considerando que el valor de cobre total para la estación 3 en noviembre fue de $5,76 \mu \mathrm{mol} / \mathrm{L}$ y el de cobre disuelto de $3,95 \mu \mathrm{mol} / \mathrm{L}$, el valor de $\mathrm{IC}_{50}=18,91 \%$ obtenido para la muestra, representaría una concentración nominal de cobre total $1,09 \mu \mathrm{mol} / \mathrm{L}$ respecto a la concentración de cobre presente en ese punto de muestreo, y un valor de cobre disuelto de $0,75 \mu \mathrm{mol} / \mathrm{L}$. Mientras que en septiembre el valor de cobre total fue de $4,8 \mu \mathrm{mol} / \mathrm{L}$ y el de cobre disuelto fue de $2,88 \mu \mathrm{mol} / \mathrm{L}$, el valor de $\mathrm{IC}_{50}=17,99 \%$ obtenido para la muestra, representaría una concentración de cobre total $0,86 \mu \mathrm{mol} / \mathrm{L}$ respecto a la concentración de cobre presente en ese punto de muestreo, y un valor de cobre disuelto de 0,52 $\mu \mathrm{mol} / \mathrm{L}$. Estos niveles se corresponden con los registrados en ensayos de laboratorio que señalan un $\mathrm{IC}_{50}$ de $0,094-6,9 \mu \mathrm{mol} / \mathrm{L}$ de cobre $^{6,7}$.

En mayo y septiembre el $\mathrm{pH}$ y cobre disuelto presentaron una correlación significativa, además las estaciones que presentaron niveles de pH más ácido fueron las estaciones 3 y 6 , coincidiendo con niveles de cobre disuelto más elevados. Esto podría explicarse porque al disminuir el pH aumenta la sorción, incrementando las concentraciones de las fracciones disuelta y biodisponible del metal ${ }^{2}$.

Un factor que no fue medido en este estudio, pero que podría haber contribuido a reducir la toxicidad de los metales en las estaciones con mayor influencia urbana, es el carbono orgánico disuelto. Este parámetro puede ser especialmente importante en la evaluación de toxicidad de cobre, ya que los complejos orgánicos actúan reduciendo la toxicidad del $\operatorname{metal}^{7,9,11}$.

La temperatura es un factor que condiciona la toxicidad, sin embargo, su acción se produce desde otro punto de vista. A diferencia del factor anteriormente mencionado, que actúa sobre la biodisponibilidad de los metales, la temperatura influencia el metabolismo biológico, incluyendo las actividades de defensa enzima - dependientes contra los efectos de contaminantes ${ }^{23}$. A pesar de la importancia biológica de este parámetro en los sistemas naturales, tanto la tasa de crecimiento $\mathrm{k}$ como la densidad celular no presentaron correlación significativa con la temperatura de la estación de muestreo. Esto puede explicarse porque durante los bioensayos se controló la temperatura, manteniéndose constante y uniforme para todos los tratamientos. De esta manera, el efecto que podría producir en la toxicidad al actuar sobre la microalga se ve alterado siendo además, teóricamente el mismo para los tratamientos de las diferentes estaciones de muestreo al tener éstos la misma temperatura durante los bioensayos.

Por otra parte, la baja correlación entre los metales pesados y la tasa de crecimiento, excepto en el mes de septiembre, podría deberse a la presencia del ácido etilendiaminotetraacetico (EDTA) disódico dihidrato en el medio de prueba, ya puede afectar la acción de los metales sobre el organismo de prueba, debido a que es un agente quelante que produce complejos, relativamente no tóxicos para las células, con muchos metales como el aluminio, bario, cadmio, cobalto, cobre, hierro, plomo, manganeso $(+2)$, níquel, estroncio y zinc reduciendo su toxicidad. Por lo tanto, es importante considerar la presencia de éste agente quelante dentro de la formulación del medio de prueba que es utilizado para los bioensayos de toxicidad ${ }^{4,7,24,25}$. En el presente estudio se utilizó este agente en la composición del medio de cultivo, lo que podría haber reducido la acción tóxica de los iones libres de los metales pesados sobre las microalgas.

\section{CONCLUSIONES}

Las aguas superficiales del río Aconcagua consideradas en este estudio pudieron estar influenciadas por la actividad minera dada 
por el incremento en las concentraciones de los metales pesados, especialmente cobre y molibdeno que estuvieron sobre la norma chilena de calidad de aguas. Se observó toxicidad en estaciones que presentaron altas concentraciones de cobre y molibdeno. Sólo en algunos períodos se observó una correlación entre la respuesta de $P$. subcapitata y las concentraciones de cobre en sus diferentes fracciones químicas, molibdeno y zinc totales, por lo que la medición sólo de las concentraciones de los metales no permitiría predecir la respuesta de $P$. subcapitata. Debido a la escasez de datos sobre la aplicación de bioensayos microalgales en ríos de Chile, los resultados obtenidos proveen valiosa información complementaria para evaluar y comprender de mejor forma los impactos de la actividad minera local sobre la calidad de las aguas superficiales.

\section{AGRADECIMIENTOS}

Esta investigación fue financiada por el proyecto Dipuv 06/2003 de la Dirección de Investigación de la Universidad de Valparaíso.

\section{REFERENCIAS}

1. O'farrel, I.; Lombardo, R.; De Tezanos, P.; Loez, C.; Environ. Poll. 2002, 120, 207.

2. Baker, S.; Herrchen, M.; Kund-rinke, K.; Klein, W.; Kördel, W.; Peijnenburg, W.; Rensing, C.; Ecotoxicol. Environ. Saf. 2003, 56, 6.

3. Walsh, G.; Yoder, M.; Laughlin, L.; Lores , E.; Ecotoxicol. Environ. Saf. 1987, 4, 215 .
4. Rodríguez, L.; Rivera, D.; Estud. Oceanol. 1995, 14, 61.

5. Ma, M.; Zhu, W.; Wang, Z.; Witkamp, G.; Aquat. Toxicol. 2003, 63, 221.

6. Cyrino, E.; Matos, R.; Roma, F.; Chemosphere 2004, 56, 369.

7. Bossuyt, B.; Janssen, C.; Aquat. Toxicol. 2004, 68, 61.

8. Koukal, B.; Guéguen, C.; Pardos, M.; Dominik, J.; Chemosphere 2003, 52 , 953.

9. Meador, J.; Sibley, T.; Swatzman, G.; Taub, F.; Aquat. Toxicol. 1998, 44, 69.

10. Moreira-Santos, M.; Soares, A.; Ribeiro, R.; Ecotoxicol. Environ. Saf. 2004, 59,164 .

11. Kramer, K.; Jak, R.; van Hattum, B.; Hooftman, R.; Environ.Toxicol.Chem. 2004, 23, 12.

12. DGA; Diagnostico y clasificación de los cursos y cuerpos de agua según objetivos de calidad, Cuenca del río Aconcagua, 2004.

13. Chile - Instituto Nacional de Normalización; NCh 411/6, Of1998, 1998.

14. The Analist; Instruction manual Orbeco-Hellige Model 975-MP; Water analysis sistem.

15. Chile - Instituto Nacional de Normalización; NCh 2706, Of2002, 2002.

16. Chile - Instituto Nacional de Normalización; NCh 1333, Of78, 1987.

17. Guevara, S.; Oyarzún, J.; Maturana, H.; Agric. Téc. 2006, 66, 57.

18. Richtera, P.; Segue, R.; Ahumada, I.; J. Chil. Chem. Soc. 2004, 49, 333.

19. SAG; Consolidado del programa de prevención y contaminación de los recursos hídricos año 1999, 2001.

20. Romero, J.; Calidad del agua. $2^{\mathrm{a}}$ ed., Alfaomega: México, 1999.

21. Universidad de Chile, Instituto de Asuntos Publicos. Informe País; Estado del Medio Ambiente en Chile. $1^{\mathrm{a}}$ ed., LOM Ediciones, 2002.

22. Franklin, N.; Stauber, J.; Apte, S.; Lim, R.; Environ. Toxicol. Chem. 2002 21,742 .

23. Eggen, R.; Behre, R.; Burkhardt-Holm, P.; Escher, B.; Schweigert, N.; Environ. Sci Technol. 2004, 38, 58 .

24. Muyssen, B.; Janssen, C.; Chemosphere 2001, 45, 507.

25. Guéguen, C.; Koukal B.; Dominio, J.; Pardos, M.; Chemosphere 2003, 53, 927. 